\title{
Climate hypersensitivity to solar forcing?
}

\author{
W. Soon ${ }^{1,2}$, E. Posmentier ${ }^{3}$, S. Baliunas ${ }^{1}$ \\ ${ }^{1}$ Harvard-Smithsonian Center for Astrophysics, 60 Garden Street, Cambridge, Massachusetts 02138, USA \\ ${ }^{2}$ Also affiliated with the Faculty of Science and Environmental Studies, University Putra Malaysia, Serdang 43400, Selangor, Malaysia \\ ${ }^{3}$ Departments of Physics and Mathematics, Long Island University, Brooklyn, New York 11201, USA
}

Received: 14 October 1999 / Revised: 16 February 2000 / Accepted: 24 February 2000

\begin{abstract}
We compare the equilibrium climate responses of a quasi-dynamical energy balance model to radiative forcing by equivalent changes in $\mathrm{CO}_{2}$, solar total irradiance $\left(S_{t o t}\right)$ and solar UV $\left(S_{U V}\right)$. The response is largest in the $S_{U V}$ case, in which the imposed UV radiative forcing is preferentially absorbed in the layer above $250 \mathrm{mb}$, in contrast to the weak response from global-columnar radiative loading by increases in $\mathrm{CO}_{2}$ or $S_{t o t}$. The hypersensitive response of the climate system to solar UV forcing is caused by strongly coupled feedback involving vertical static stability, tropical thick cirrus ice clouds and stratospheric ozone. This mechanism offers a plausible explanation of the apparent hypersensitivity of climate to solar forcing, as suggested by analyses of recent climatic records. The model hypersensitivity strongly depends on climate parameters, especially cloud radiative properties, but is effective for arguably realistic values of these parameters. The proposed solar forcing mechanism should be further confirmed using other models (e.g., general circulation models) that may better capture radiative and dynamical couplings of the troposphere and stratosphere.
\end{abstract}

Key words: Meteorology and atmospheric dynamics (climatology, general or miscellaneous) -

Solar physics, astrophysics, and astronomy

(ultraviolet emissions)

\section{Introduction}

About $54 \%$ of the decadal- and longer-scale variations of the worldwide-averaged land surface temperature

Correspondence to: W. Soon

e-mail: wsoon@cfa.harvard.edu variance $^{1}$ over the last 100 years can be optimally attributed to intrinsic solar total irradiance change, and $38 \%$ to effects of increased anthropogenic greenhouse gases (GHGs), leaving a small $8 \%$ unexplained $^{2}$ (e.g., Soon et al., 1996). But the inferred solar radiative forcing change (of $0.5 \%$ in $S_{\text {tot }}$; see also Baliunas and Jastrow, 1990; Hoyt and Schatten, 1993), slightly less than $1 \mathrm{~W} \mathrm{~m}^{-2}\left(0.5 \%\right.$ of $S_{\text {tot }}$ converted into forcing at the top of atmosphere), is significantly smaller than the estimated global forcing of $2.4 \pm 0.4 \mathrm{~W} \mathrm{~m}^{-2}$ resulting from the increases of the anthropogenic GHG concentrations over the last 100 years (e.g., IPCC, 1996). There is thus an apparent inconsistency between weak solar radiative forcing and large climatic response. Furthermore, if the long recovery times that are implied by model experiments of the series of late nineteenth and early twentieth century volcanic cooling events can be used to rule against the possibility of a large equilibrium climate sensitivity (Lindzen and Giannitsis, 1998), then the acuteness of the apparent sun-climate inconsistency is exacerbated. However, there are several ways in which this discrepancy might be resolved.

First, it might be argued that the solar radiative forcing of about $1 \mathrm{~W} \mathrm{~m}^{-2}$ and the response of $0.27^{\circ} \mathrm{C}$ $\left(54 \%\right.$ of the total observed warming of about $\left.0.5^{\circ} \mathrm{C}\right)$ are consistent with the IPCC range of climate sensitivity, so the observed climate response to solar forcing is not enigmatic. However, a different sensitivity would be necessary to reconcile the anthropogenic greenhouse forcing of $2.4 \mathrm{~W} \mathrm{~m}^{-2}$ with the response

\footnotetext{
${ }^{1}$ These long-term temperature variances are based on the 11 year running mean record of the annual-mean, worldwide-averaged land temperature taken from NASA GISS database.

${ }^{2}$ The numbers $54 \%$ and $38 \%$ are the individual $71 \%$ and $51 \%$ of explained variances associated with the solar and GHG forcings, respectively, (case D3 of Soon et al., 1996, p. 897) scaled as follows in order to sum to a total of $92 \%$ of explained variance (case D3) as follows:
}

(a) Solar Part: $(71 /[51+71]) \times 92 \%=54 \%$

(b) GHG Part: $(51 /[51+71]) \times 92 \%=38 \%$ 
of $0.19{ }^{\circ} \mathrm{C}\left(38 \%\right.$ of $\left.0.5{ }^{\circ} \mathrm{C}\right)$, so the seeming contradiction reappears. ${ }^{3}$

A second way to resolve the apparent contradiction in terms of the strictly thermodynamical responses of the climatic system to 'externally' imposed radiative forcings would be to invoke other, as yet undetermined, radiative forcings negatively correlated with GHG forcing. One example is an incomplete accounting of aerosols in the radiative budget of the climate system, suggested by the new estimate of the direct forcing of $-1.6 \pm 0.5 \mathrm{~W} \mathrm{~m}^{-2}$ from 48 components of natural and anthropogenic aerosols (Jacobson, 1999; see also Haywood et al., 1999 for further discussion on the important contribution by naturally occurring sea-salt aerosols for the total tropospheric aerosol forcing).

A third type of resolution is possible if the sunclimate connection were viewed through the perspective of climate dynamics, in which the simple thermodynamics of direct radiative heating of the terrestrial atmosphere and surface is not the sole mechanism capable of yielding significant responses. For example, Wallace et al. (1995) argued that about half of the temporal variance of the monthly mean Northern Hemisphere surface air temperature during the period 1900-1990 is dynamically produced by a change in the atmospheric circulation connected to a distinctive pattern of anomalously cold ocean and warm land. Furthermore, such dynamics can cause unforced, internal climate variability that might be difficult to distinguish from externally forced variability or might modify the response to external forcing (e.g., Palmer, 1999; Posmentier et al., 1999 and references therein).

So strictly speaking, this third type of resolution may be subdivided into (a) purely internal natural variability; and (b) mechanisms involving the external triggering of internally-determined climate shifts, or external forcing amplified by internal feedback mechanisms.

Subtype (a) of the third category of resolutions would invoke the possibility of purely internal natural variability that might correlate by coincidence with solar variability. This is because studies such as Soon et al. (1996; including several other references cited therein) generally cannot validate or invalidate causality among correlated variables. Thus, it cannot be ruled out that the part of climate variability that correlates with solar variability is actually an unforced internal variability with a coincidental correlation with the solar forcing variability. Alternatively, a robust mechanism that can serve to trigger or amplify (or both, i.e., resolution subtype $3 b$ ) the response to changes in the external solar

\footnotetext{
${ }^{3}$ Although the difference of the estimated solar and GHGs radiative forcings appears to be real, it would certainly be imprudent to overlook the warning by Sinha and Harries (1997) and Forster et al. (1997) that radiative forcing should not be used as the lone indicator of possible climate change (In fact, our independent study here yields this similar caution). The caution arises from the demonstration by Sinha and Harries (1997), mainly through differences in spectral responses, that substantial changes in heating and cooling of the atmosphere and surface are possible under a scenario of the near-cancellation of net radiative forcing with simultaneous tropospheric aerosol and $\mathrm{CO}_{2}$ perturbations.
}

radiative or corpuscular (or both) forcing ${ }^{4}$ would also resolve the apparent inconsistency between weak solar forcing and large climate response.

There are several examples of feedback mechanisms in subtype (b) of the third category of resolutions of the discrepancy. Fowler and Randall (1994) proposed a powerful global negative feedback involving the formation of upper-tropospheric stratiform clouds, atmospheric radiative cooling (ARC) and convective activity. They conceived of an external perturbation that forces more vigorous convection (initiated, say, through enhanced surface ocean warming), more upper tropospheric cloudiness, hence less ARC and more static stability, which ultimately couples back to reduce convective activity. The large effect of ice clouds in enhancing atmospheric static stability has been confirmed and clarified in simulations of Ho et al. (1998). Other promising coupling mechanisms studied so far include solar UV forcing change (e.g., Kodera et al., 1991; Rind and Balachandran, 1995) and stratospheric ozone change (e.g., Haigh, 1999; Shindell et al., 1999), both of which have been shown to be capable of amplifying solar irradiance forcing by modulation of both the amount and distribution of upward-propagating planetary waves which then cause significant changes to the circulation patterns of the low and middle atmospheres (see also Arnold and Robinson, 1998).

However, none of these mechanisms has been generally accepted as adequate to resolve the apparent sunclimate inconsistency, because of a lack of either theoretical proof of robustness or empirical studies resolving the effects of individual mechanisms. At this time, it remains possible that climatic change caused by solar variability is modulated mainly by only one of these mechanisms, by a combination of more than one of them, or by mechanisms still not identified. Furthermore, it may not be possible to identify unambiguously the relative roles of these mechanisms.

The purposes of this work are (1) to suggest an alternative mechanism whereby solar variability can cause a disproportionately large climate response, (2) to estimate the empirically testable ratios among changes in observable climate variables which would occur according to this mechanism, and (3) to show that this mechanism's underlying assumptions are plausible, and that it may be a viable candidate for the explanation of the weak-solar-forcing/large-climate-response inconsistency. Briefly, the proposed amplification mechanism works by having solar UV irradiance (wavelengths less than $0.4 \mu \mathrm{m}$ ) heat the lower stratosphere and upper troposphere, and significantly modulate the amount of upper-atmospheric convection, ice-cloud cover and ozone concentration, which consequently alter the transparency of the terrestrial atmosphere to solar shortwave $(\mathrm{SW})$ radiation.

\footnotetext{
${ }^{4}$ We shall restrict discussion to intrinsic change in the solar radiative output. See e.g., Tinsley (1996) and Soon et al. (2000) for additional, possible modulation mechanisms related to effects of solar and cosmic-ray charged particles.
} 


\section{Description of the climate model}

The model used to calculate climate response has seven zones of latitude, with land and sea regions in each. The seven zones comprise a polar convergence zone, a temperate divergence zone and a subtropical divergence zone in each hemisphere, and one intertropical convergence zone. Each of the 14 regions has four atmospheric layers: low (1000-700 mb), mid (700-400 mb), high (400-250 mb) and 'upper-troposphere/stratosphere' $(<250 \mathrm{mb})$ and one surface layer, with a homogeneous, deep ocean. (Note that throughout the text, the model terms 'stratosphere' and 'upper-level clouds' refer to phenomena associated with the top-most layer of our model.)

The equilibrium values of temperature, precipitation and cloud content of each atmospheric cell are simultaneously computed for 256 times per year, based on the conservation equations for water and energy, taking into account three-dimensional advection and mixing, sensible and latent heat, and radiation. The rates of change of the surface and deep ocean temperatures based on these atmospheric equilibrium values are integrated using an Adams-Bashforth fourth-order scheme. Each process rate is computed as described below from functions using either constants directly based on observation, or free parameters determined by tuning the model.

The radiation scheme uses seven pseudo-bands. These are not bands of contiguous wavelengths. Rather, each is an aggregate of radiation with various wavelengths but with consistent radiative properties: a 'black' infrared band, a 'transparent' infrared band, a 'solar' band and four 'gray' infrared bands in which absorption is the results of either $\mathrm{O}_{3}, \mathrm{H}_{2} \mathrm{O}, \mathrm{CO}_{2}$, or both $\mathrm{H}_{2} \mathrm{O}$ and $\mathrm{CO}_{2}$. The fractional energy in each band and the dependence of optical thickness on gas concentration, are based on Ellingson et al. (1991).

The atmospheric general circulation is modulated by the meridional gradients of geopotential height, with one constant determined by observations (Peixoto and Oort, 1992). This allows the general circulation to vary both seasonally and in response to interannual climate change, thus including an important feedback in the model. Similarly, a Walker-type zonal circulation, landward below $400 \mathrm{mb}$ and seaward above when the land is warmer than the ocean (or vice versa) is the same function of the zonal gradients of geopotential height. At 700 and $400 \mathrm{mb}$, the vertical mixing rate is an ad hoc function of the Richardson's number, with two constants determined by the observed dependence. At $250 \mathrm{mb}$, the vertical mixing is treated as upward penetrative convection. This is implemented by assuming a vertical mixing rate proportional to a quadratic function of the tropopause height, while the tropopause height, in turn, is an ad hoc function of stability with two constants determined by the results of Thuburn and Craig (2000). (Tropopause height in this model is obviously not resolved by the temperatures of the four layers; it is only a figurative intermediary used to compute convective mixing at $250 \mathrm{mb}$ from atmo- spheric stability.) In this initial study, we have not included the important effect of lower stratospheric quasi-biennial-oscillation (QBO) of equatorial zonal winds.

In the ocean, the meridional mixing rate, and the vertical mixing rate between the cold water sphere and the warmer surface layer regions, are both observationally determined. In addition, surface water sinks poleward of a specified isotherm $\left(2.0^{\circ} \mathrm{C}\right.$ in the northern polar region, and $-0.5^{\circ} \mathrm{C}$ in the southern polar region), at a rate which causes cold water formation at an annual-mean rate of $44 \mathrm{~Sv}$. To conserve mass, upwelling at a constant rate throughout the ocean balances the high latitude sinking rate.

The surface evaporation rate is computed from the surface temperature and the low-layer humidity using one constant determined by observed global mean evaporation. Similarly, the sensible heat flux from the surface to the atmosphere is computed from the surface and low-layer temperatures using one constant determined by observations and one free parameter. The cloudiness and the precipitation for each atmospheric cell are computed from the temperature, specific humidity and the mean and convective circulation in the cell using two constants determined by observations and two free parameters.

In order to tune and verify the model (see e.g., Soon et al., 1996), the computed land and sea values in all seven regions of the summer, winter, and annual average surface temperature and evaporation; the temperature, precipitation and cloud area within each layer; all the components of surface and atmospheric energy balance; snow and ice areas; and the deep sea temperature and deep water formation rate were compared with observations. If at least three of these variables are independent in at least half of the model's 14 regions, at least once in time, the model results would have at least 21 degrees of freedom. On the other hand, only the three free parameters mentioned may be adjusted to produce agreement between the model and observations. Thus, the model is robustly over-determined. In addition, the model has been used to simulate successfully the climate's response to Milankovic forcing and nonlinear dynamical behaviour including abrupt climate shifts and chaotic variabilities (e.g., Posmentier et al., 1999).

\section{Climate experiments}

We performed a total of four experiments: one reference run together with three contrasting radiative perturbation experiments.

In the reference climate, the upper-level clouds are assigned to high reflectivity in order to simulate the observed properties of the tropical thick cirrus ice cloud system (e.g., Ramaswamy and Ramanathan, 1989; Heymsfield et al., 1998). To represent, albeit crudely, the effects of ice clouds, we simply prescribed an elevated mean SW scattering albedo of 0.8 for those upper-level clouds uniformly across all zones. In addi- 
tion, we have also added runs that selectively assigned only high value of upper-level cloud reflectivity of 0.8 aloft the Inter-Tropical Convergence and North/South Dry Subtropical zones while keeping the upper-level cloud reflectivity to a lower value of 0.18 above the North/South Wet Temperate and Polar zones. Detailed microphysical cloud studies, such as that by Ho et al. (1998), are able to attribute the property of high reflectivity in ice cirrus (over those of water clouds) to a much larger particle size, a smaller extinction coefficient and a generally smaller wavelength-dependent asymmetry factor. It is also useful to clarify that in such a thick-cloud system, the solar SW-albedo effects dominate the longwave (LW)-trapping greenhouse effects such that the net cloud radiative LW-SW flux is always negative, in contrast to the more commonly discussed LW-effect dominant in thin cirrus (e.g., see the contrasting examples in Fig. 2 of Heymsfield et al., 1998).

The three perturbed runs are as follows:

1. Equivalent $\mathrm{CO}_{2}$ [or $\left.1.2 \times\left(\mathrm{CO}_{2}\right)_{\text {ref }}\right]$ case: the wellmixed atmospheric $\mathrm{CO}_{2}$ is increased from 350 to 420 ppm, which causes a $1.0 \mathrm{~W} \mathrm{~m}^{-2}$ (i.e., pure radiative forcing without the influence of climatic feedbacks) reduction of the net outgoing IR at $250 \mathrm{mb}$.

2. Equivalent $S_{\text {tot }}$ case: the solar constant is increased by $0.5 \%$, which causes a $1.0 \mathrm{~W} \mathrm{~m}^{-2}$ increase in the net down-going solar radiation at $250 \mathrm{mb}$ exactly equal to the increased trapping of outgoing IR at $250 \mathrm{mb}$ by increasing $\mathrm{CO}_{2}$ to $420 \mathrm{ppm}$.

3. Equivalent $s_{U V}$ case: solar radiation of $1.0 \mathrm{~W} \mathrm{~m}^{-2}$ is selectively absorbed in the model 'stratosphere' and global stratospheric $\mathrm{O}_{3}$ increases by $5.4 \%$ as suggested by 2-D photochemical model calculations or extrapolation from observations (e.g., Wuebbles et al., 1998; Haigh, 1999). This solar 'equivalence' scenario represents the increased emission of the solar UV irradiation and enhanced heating that also included the absorption of near-IR and visible solar fluxes by thick cirrus cloud system and increased stratospheric $\mathrm{O}_{3}$ (e.g., Ramaswamy and Ramanathan, 1989).

\section{Discussion}

The main results of the three perturbed experiments are summarized in Table 1. Roughly similar responses were obtained in the first two perturbed runs, where the radiative energy is 'column-loaded' (absorbed in all four atmospheric layers), with a $20 \%$ smaller change in surface temperature $\left(T_{s f c}\right)$ for the $1.2 \times\left(\mathrm{CO}_{2}\right)_{\text {ref }}$ case compared to the equivalent $S_{t o t}$ case.

Before proceeding to compare the results of the first two perturbed runs with the response of the equivalent $S_{U V}$ case, it might be noted that in the increased $\mathrm{CO}_{2}$ experiment, a modest increase in 'stratospheric' temperature is observed, while there are both theoretical and empirical reasoning to support that a $\mathrm{CO}_{2}$ increase should cause cooling above $100 \mathrm{mb}$ (e.g., Forster and Shine, 1999). However, warming of the average temperature above $250 \mathrm{mb}$ is not necessarily inconsistent with cooling of the upper $40 \%$ of that layer (i.e., above 100 $\mathrm{mb}$ ). Obviously, our four-layer model atmosphere cannot resolve this effect, so it is neither consistent nor inconsistent with higher-resolution models on this point. However, the underlying physics of changes in water vapor affecting optical thickness and the subsequent effect on radiation balance and temperature is represented in our model, so the cooling effect above $100 \mathrm{mb}$ is implicitly averaged into the changes in our 'stratospheric' temperature. A more pertinent reason that our model's inability to resolve changes above $100 \mathrm{mb}$ may not be critical to our overall results is that the feedbacks essential to our proposed mechanism (under the equivalent $S_{U V}$ forcing scenario) hinge on changes in the vertical temperature difference between the 'high' and 'stratospheric' layers, not on the individual temperatures. In a $\mathrm{CO}_{2}$ doubling experiment (not included in Table 1), we find that this vertical temperature difference decreases by $0.7^{\circ} \mathrm{C}$ over $7 \mathrm{~km}$, which compares well with the approximately $0.5^{\circ} \mathrm{C}$ over $5 \mathrm{~km}$ in the layer around $250 \mathrm{mb}$ calculated by GCM (e.g., Hansen et al., 1997, Fig. 4b). (Note that the vertical distance from midpressure of the 'stratosphere' layer, $125 \mathrm{mb}$, to the midpressure of the 'high' layer, $325 \mathrm{mb}$, is $7 \mathrm{~km}$.)

Table 1. Annual-mean parameters: ${ }^{\mathrm{a}}$ intercomparison of responses of $\mathrm{CO}_{2}, S_{t o t}$ and $S_{U V}$ forcings

\begin{tabular}{llllllll}
\hline Experiment & $\begin{array}{l}T_{s f c} \\
\left({ }^{\circ} \mathrm{C}\right)\end{array}$ & $\begin{array}{l}T_{s t} \\
\left({ }^{\circ} \mathrm{C}\right)\end{array}$ & $\begin{array}{l}\Delta T_{\text {lat }} \\
\left({ }^{\circ} \mathrm{C}\right)\end{array}$ & $\begin{array}{l}q_{s t} \\
(\mathrm{ppmv})\end{array}$ & $K_{250}$ & $R_{\text {stCld }}$ & snice \\
\hline Reference $^{\mathrm{b}}$ & 13.5 & -64.4 & 32.7 & 88.8 & 1.00 & 0.109 & 0.139 \\
$1.2 \times\left(\mathrm{CO}_{2}\right)_{\text {ref }}{ }^{\mathrm{c}}$ & 14.5 & $-63.7^{\mathrm{d}}$ & 31.3 & 94.4 & 1.00 & 0.109 & 0.125 \\
Equivalent $S_{\text {tot }}{ }^{\mathrm{c}}$ & 14.7 & $-63.6^{\mathrm{d}}$ & 31.3 & 96.8 & 1.00 & 0.110 & 0.124 \\
Equivalent $S_{U V}{ }^{\mathrm{c}}$ & 16.5 & $-58.7^{\mathrm{d}}$ & 29.2 & 77.4 & 0.68 & 0.096 & 0.100 \\
\hline
\end{tabular}

${ }^{a}$ Parameters: $T_{s f c}$ global surface temperature; $T_{s t}$ global temperature above $250 \mathrm{mb} ; \Delta T_{\text {lat }}$ mean tropic-to-pole surface temperature gradient; $q_{s t}$ global $\mathrm{H}_{2} \mathrm{O}$ mixing ratio above $250 \mathrm{mb} ; K_{250}$ global diffusive vertical transport index across the model $250 \mathrm{mb}$ interface; $R_{\text {stCld }}$ contribution of 'upper-level cloud' to SW reflectance; snice global fractional snow/ice cover; albedo planetary albedo

b The reference climate state with $350 \mathrm{ppm}$ of $\mathrm{CO}_{2}$ and solar total irradiance, $S_{\text {tot }}$, of $1370 \mathrm{Wm}^{-2}$

${ }^{\mathrm{c}}$ See text for description d Our model's upper-most layer cannot resolve the expected cooling above $100 \mathrm{mb}$ or so (e.g., see Forster and Shine, 1999) from increased $\mathrm{CO}_{2}$ and $\mathrm{H}_{2} \mathrm{O}$ concentrations in the stratosphere (arising from either a direct radiative forcing or a radiative-induced feedback). But the observed qualitative behavior for the hypersensitive response for the equivalent $S_{U V}$ scenario (relative to the $1.2 \times\left(\mathrm{CO}_{2}\right)_{\text {ref }}$ and equivalent $S_{\text {tot }}$ cases) seems robust over the perturbed forcing amplitudes ranging 0.5 to $7 \mathrm{Wm}^{-2}$ (see text for further discussion) 
Table 1 shows that a drastically different response was obtained in the equivalent $S_{U V}$ forcing case. The response is about three times larger than in the columnloaded cases. In the $S_{U V}$ forcing case, the warmer 'stratosphere' $\left(T_{s t}\right)$ causes an increase in upper-atmospheric stability, which reduces the convective flux of moisture, dries the upper atmosphere $\left(q_{s t}\right)$ and reduces the SW energy loss by 'upper-level clouds' reflection (proportional to $R_{s t C l d}$ in Table 1). Those effects, in turn, allow more incoming solar SW radiation to warm the surface and to modulate the snow/ice cover (snice) and decrease the planetary albedo. This way of heating the upper atmospheric layer also taps into the cloud-surface snow/ice albedo coupling feedback. Changes in convective activity and in large-scale circulation dynamics would obviously occur as a result of changes in the vertical transport coefficient $\left(K_{250}\right)$ and the gradient of the tropic-to-pole surface temperature $\left(\Delta T_{\text {lat }}\right)$.

To study the robustness of the amplification seen in the equivalent $S_{U V}$ experiment, we performed additional runs to study the dependence of the results on the amplitude of radiative forcings (in the range of 0.5 to $7 \mathrm{~W} \mathrm{~m}^{-2}$ ) and upper-level cloud properties. There are two preliminary conclusions. First, the amplification effects were observed for all the forcing amplitudes studied. Second, as expected, the high SW scattering albedo for upper-level clouds is very important. That result comes from calculation of the responses for values of upper clouds scattering albedos in the range 0.1 (more closely approximating water clouds) to 0.9. Only cases with scattering albedo larger than 0.7 or so manifest climatic amplification in response to imposed solar UV forcings. While such a restriction is not contradictory to current understanding of cloud physics (e.g., Heymsfield et al., 1998), it does draw attention to the sensitivity of the mechanism proposed here to arguable but uncertain cloud radiative properties assumed in our experiments, and to the need for research on cirrus, cirrostratus, cirrocumulus, and tops of cumulonimbus (with anvil) clouds over the tropics and mid-latitudes (see e.g., Chen et al., 2000). Thus, the amplification found for the equivalent $S_{U V}$ case appears to operate only as long as the important solar UV heating-upper-level cloud feedback is in effect. Further confirmation of the mechanism is necessary by examining responses according to other climate models including general circulation models.

Another important test for the amplification mechanism seen in the equivalent $S_{U V}$ comes from assigning only a high value of upper-level cloud reflectivity, 0.8 , aloft the InterTropical Convergence and North/South Dry Subtropical zones in our model while keeping the upper-level cloud reflectivity to a lower value, 0.18, above the North/South Wet Temperate and Polar zones. We confirm the qualitative behavior of the atmospheric response seen for the results shown in Table 1.

The last, additional comparison run (not shown), with only a change in the stratospheric $\mathrm{O}_{3}$ concentration, suggests that direct stratospheric $\mathrm{O}_{3}$ IR radiative forcing has only a minor effect.
The key points of our proposed climate hypersensitivity mechanism are:

1. The Sun is more variable in the UV than in the visible bands. However, the increased UV irradiance is mainly absorbed in the lower stratosphere/upper troposphere rather than at the surface.

2. Absorption in the stratosphere raises the temperature moderately around the vicinity of the tropopause, and tends to stabilize the atmosphere against vertical convective/diffusive transport, thus decreasing the flux of heat and moisture carried upward from surface.

3. The decrease in the upward convection of heat and moisture tends to raise the surface temperature because a drier upper atmosphere becomes less cloudy, which in turn allows more solar radiation to reach the Earth's surface.

This top-to-bottom route of upper atmospheric solar heating may be an efficient mechanism of amplifying lower atmospheric responses to radiative perturbations in the stratosphere. Such a solar mechanism may also be potentially interesting for study of past climate change without confusion from anthropogenic forcing. The potentially synergistic coupling of this independent, solar UV irradiance mechanism, along with changes in total irradiance (whose energetics are concentrated in the visible) or with any other physical mechanism (like the modulation heat storage of the upper surfaces of the World Ocean, White et al., 1997) may only be revealed with time-dependent experiments constrained by observations.

Acceptance of the proposed hypersensitivity mechanism based on coupling among solar UV forcing, atmospheric static stability, upper-level clouds and $\mathrm{O}_{3}$ would require two confirmatory results. First, a determination of upper-cloud radiative properties consistent with those assumed in our experiments is necessary. Second, GCMs and other models should be used to scrutinize further the robustness of the mechanism suggested by our semi-empirical results for the climate hypersensitivity to solar variability. Such experimentation would study the possible interactions of our mechanism with the internal dynamical phenomena like ENSO (El Nino-Southern Oscillation), NAO (North Atlantic Oscillation) and/or AO (Arctic Oscillation) as well as the stratospheric QBO (Palmer, 1999; Hartmann et al., 2000 and references therein).

We conclude that it is useful to recognize the Sun as a variable, multiwavelength emitter which may be capable of significant modulation of terrestrial atmospheric dynamics (i.e., climate) through more than one of diverse physical coupling channels.

Acknowledgements. This work was supported by the Electric Power Research Institute. Additional support came from the National Aeronautics and Space Administration (Grant NAG57635). E. S. P. further acknowledges the support of Long Island University Research Release Time program. We are grateful to constructive comments of an anonymous reviewer.

Topical Editor J.-P. Duvel thanks H. Le Treut and R. Kandel for their help in evaluating this paper. 


\section{References}

Arnold, N. F., and T. R. Robinson, Solar cycle changes to planetary wave propagation and their influence on the middle atmospheric circulation, Ann. Geophysicae, 16, 69-76, 1998.

Baliunas, S., and R. Jastrow, Evidence for long-term brightness changes of solar-type stars, Nature, 348, 520-523, 1990.

Chen, T., W. B. Rossow, and Y.-C. Zhang, Radiative effects of cloud-type varitions, J. Clim., 13, 264-286, 2000.

Ellingson, R. G., J. Ellis, and S. Fels, The intercomparison of radiation codes used in climate models: longwave results, J. Geophys. Res., 96, 8929-8953, 1991.

Forster, P. M. de F., and K. P. Shine, Stratospheric water vapour changes as a possible contributor to observed stratospheric cooling, Geophys. Res. Lett., 26, 3309-3312, 1999.

Forster, P. M. de F., R. S. Freckleton, and K. P. Shine, On aspects of the concept of radiative forcing, Clim. Dyn., 13, 547-560, 1997.

Fowler, L. D., and D. A. Randall, A global radiative-convective feedback, Geophys. Res. Lett., 21, 2031-2038, 1994.

Haigh, J. D., A GCM study of climate change in response to the 11-year solar cycle, Q. J. R. Meteorol. Soc., 125, 871-892, 1999.

Hansen, J., M. Sato, and R. Ruedy, Radiative forcing and climate response, J. Geophys. Res., 102, 6831-6864, 1997.

Hartmann, D. L., J. M. Wallace, V. Limpasuvan, D. W. J. Thompson, and J. R. Holton, Can ozone depletion and global warming interact to produce rapid climate change? Proc. Natl. Acad. Sci. (USA), 97, 1412-1417, 2000.

Haywood, J. M., V. Ramaswamy, and B. J. Soden, Tropospheric aerosol climate forcing in clear-sky satellite osbervations over the oceans, Science, 283, 1299-1303, 1999.

Heymsfield, A. J. et al., Cloud properties leading to highly reflective tropical cirrus: interpretations from CEPEX, TOGA, COARE, and Kwajalein, Marshall Island, J. Geophys. Res., 103, 8805-8812, 1998.

Ho, C.-H., M.-D. Chou, M. Suarez, and K.-M. Lau, Effect of ice cloud on GCM climate simulations, Geophys. Res. Lett., 25, 71-74, 1998.

Hoyt, D. V., and K. H. Schatten, A discussion of plausible solar irradiance variations, 1700-1992, J. Geophys. Res., 98, 18 895$18906,1993$.

Intergovernmental Panel on Climate Change (IPCC), Climate change 1995-the science of climate change, Ed. J. T. Houghton et al., 572 pp., Cambridge University Press, New York, 1996.

Jacobson, M. Z., A study of the global direct radiative forcing due to multicomponent anthropogenic and natural aerosols, in Symposium on Interdisciplinary Issues in Atmospheric Chemistry, AMS 1999 Annual Meeting, 39-41, AMS, Dallas, Texas, 1999.
Kodera, K., M. Chiba, and K. Shibata, A general circulation model study of the solar and QBO modulation of the stratospheric circulation during the northern hemisphere winter, Geophys. Res. Lett., 18, 1209-1212, 1991.

Lindzen, R. S., and C. Giannitsis, On the climatic implications of volcanic cooling, J. Geophys. Res., 103, 5929-5941, 1998.

Palmer, T. N., A nonlinear dynamical perspective on climate prediction, J. Clim., 12, 575-591, 1999.

Peixoto, J. P., and A. H. Oort, Physics of climate, 520 pp., American Institute of Physics, New York, 1992.

Posmentier, E. S., W. H. Soon, and S. L. Baliunas, Natural variability in an ocean-atmosphere climate model, J. Fizik (Physics) Malay., 19, 157, 1999.

Ramaswamy, V., and V. Ramanathan, Solar absorption by cirrus clouds and the maintenance of the tropical upper troposphere thermal structure, J. Atmos. Sci., 46, 2293-2310, 1989.

Rind, D., and N. K. Balachandran, Modeling the effects of UV variability and the $\mathrm{QBO}$ on the troposphere-stratosphere system. Part II: the troposphere, J. Clim., 8, 2080-2095, 1995.

Shindell, D., D. Rind, N. Balachandran, J. Lean, and P. Lonergan, Solar variability, ozone and climate, Science, 284, 305-308, 1999.

Sinha, A., and J. E. Harries, Possible change in climate parameters with zero net radiative forcing, Geophys. Res. Lett., 24, 23552358, 1997.

Soon, W., S. Baliunas, E. S. Posmentier, and P. Okeke, Variations of solar coronal hole area and terrestrial lower tropospheric air temperature from 1979 to mid-1998: Astronomical forcings of change in Earth's climate? New Astron., 4, 563-579, 2000.

Soon, W. H., E. S. Posmentier, and S. L. Baliunas, Inference of solar irradiance variability from terrestrial temperature changes, 1880-1993: An astrophysical application of the Sun-climate connection, Astrophysi. J., 472, 891-902, 1996.

Tinsley, B. A., Correlations of atmospheric dynamics with solar wind-induced changes of air-earth current density into cloud tops, J. Geophys. Res., 101, 29701-29714, 1996.

Thuburn, J., and G. C. Craig, Stratospheric influence on tropopause height: The radiative constraint, J. Atmos. Sci., 57, 17-28, 2000.

Wallace, J. M., Y. Zhang, and J. A. Renwick, Dynamic contribution to hemispheric mean temperature trends, Science, 270, 780-783, 1995.

White, W. B., J. Lean, D. R. Cayan, and M. D. Dettinger, Response of global upper ocean temperature to changing solar irradiance, J. Geophys. Res., 102, 3255-3266, 1997.

Wuebbles, D. J., C.-F. Wei, and K. O. Patten, Effects on stratospheric ozone and temperature during the Maunder Minimum, Geophys. Res. Lett., 25, 523-526, 1998. 